\title{
Road Safety Surveillance and Intelligent Traffic Management System
}

\author{
Sushma S, Swathi S, Tulasi G, Vinutha M, Nandakumar A N* \\ Department of CSE, GSSSIETW, Mysuru, Karnataka, India
}

DOI: https://doi.org/10.21467/proceedings.1.3

* Corresponding author email: nandakumar@gsss.edu.in

\begin{abstract}
Road Safetyis one of the most vitalthinginourdeveloping world. This paper presents the study ofvarious aspects and issues related to the problem and it provides the solution. This paperemphasizes on using prominent technology -Internet of Things(Io'T) for developing smart system to monitor various parametersrelated to road traffic and using it for effective solution. Thesurvey of the existing systems and concerned techniques relatedto the problem area is discussed. Different issues like no-horn zones, obstacle detection, parking zones and related methods to solve these issues. We propose our "Road Safety Surveillance and Intelligent Traffic Management System" consisting of Raspberry pi which will fetch the rules from the cloud compares it with the current position which is obtained using GPS System and sends signal to the circuits to carry out the corresponding work.
\end{abstract}

Keywords - Cloud, GPS Module, IoT, No-horn Zone, Raspberry pi.

\section{Introduction}

Rising road Traffic is one of the biggest problem tackled by the world in day to day life. People suffer every day in some or the other way due to heavy road traffic. It is much important to consider and improve the factor of road safety while thinking about the problem of road traffic. The efficient solution to this problem is to use smart digital technology for handling the real time traffic. There has been various research work carried out to find the solution for this problem. But still there is a need of efficient and compatible system that can be practically tested and implemented.

Traffic surveillance can be very useful for future planning such as pollution control, traffic signal control, infrastructure and road planning, accidental blockage avoidance. The data obtained from this system can be useful for the prediction of the traveling time and path.

In the proposed system, we have 3 problems to be handled, which are discussed below. Firstly, the rulesgiven by the RTO regarding no-horn zone, parking zone is stored in the cloud by the administrator. Then the Raspberry pi embedded in the circuit of the proposed system, retrieves the data from the cloud and if it matches with the current location of the vehicle and if it's a no-horn zone, thenhorn is disabled. If it's horn-zone, then horn will be enabled. The above mechanism holds same for parking-zone, that is if the current location is a parking zone, then

\footnotetext{
(C) 2018 Copyright held by the author(s). Published by AIJR Publisher in Proceedings of the $3^{\text {rd }}$ National Conference on Image Processing, Computing, Communication, Networking and Data Analytics (NCICCNDA 2018), April 28, 2018.

This is an open access article under Creative Commons Attribution-NonCommercial 4.0 International (CC BY-NC 4.0) license, which permits any non-commercial use, distribution, adaptation, and reproduction in any medium, as long as the original work is properly cited. ISBN: 978-81-936820-0-5
} 
Road Safety Surveillance and Intelligent Traffic Management System

the driver will be noticed the present location is a parking-zone on an led display. If its not a parking-zone, it too willbe alerted to the driver on the display. Coming to the third feature, it is obstacle detection, where we divide the distance between the vehicle and the obstacle into three sections, the first one is safe zone where no action is taken, the second one is the caution zone, where caution is given to the driver, and the third zone is danger zone where the vehicle is very near to the obstacle and a buzzer is enabled to alert the driver.

\section{LITERATURE SURVEY}

Road safety is of high concern with growing world. [1]. Internet of things based smart transportation systems: According to J. Sherly1,D.Somasundareswari Scholar, ECE Department,SNS College of Technology, Tamilnadu, India.They focus on to an urban IoT system that is used to build intelligent transportation system (ITS). IoT based intelligent transportation systems are designed to support the Smart City vision, which aims at employing the advanced and powerful communication technologies for the administration of the city [2]. Accident avoidance and detection On highways: According to S.P. Bhumkar, V.V. Deotare, R.V.BabarSinhgad Institute Of Technology, Lonavala, Pune, India. They describe a real-time online safety prototype that controls the vehicle speed under driver fatigue. The purpose of such a model is to advance a system to detect fatigue symptoms in drivers and control the speed of vehicle to avoid accidents. The main components of the system consist of number of real time sensors like gas, eye blink, alcohol, fuel, impact sensors and a software interface with GPS and Google Maps APIs for location.[3].Surveillance, privacy and the ethics of vehicle Safety communication technologies:According to M. Zimmer, PhD Candidate,Department of Culture and Communication,New York University.Vehicle safety communication (VSC) technologies rely on the creation of autonomous, self-organizing, wireless communication networks connecting vehicles with roadside infrastructure and with each other [4]. Algorithm for Back-up and Authentication of Data Stored on Cloud: According to ManaliRaje, Maharashtra Institute of TechnologyPune, IndiaEveryday a huge amount of data is generated in Cloud Computing. The maintenance of this electronic data needs some extremely efficient services. There is a need to properly collect this data, check for its authenticity and develop proper backups is needed. The Objective of this paper is to provide Response Server, some solution for the backup of data and its restoration, using the Cloud. The collection of the data is to be done from the client and then the data should be sent to a central location.

\section{Proposed System}

Traditionally, road safety has been assumed to be the responsibility of the transport sector. A Safety Management System is a systematic approach to managing safety, including the necessary organizational structures, accountabilities, policies and procedures. Many in-vehicles monitoring devices are essentially with in-built accelerometers, GPS and other features. The advantage of safety surveillance system is that it provides data to show when, where and how 
a vehicle is being driven. The speed of the vehicles, no horn areas, and other details are maintained by the RTO department and can easily keep track of vehicles from this.

Our work is made up of 3 problems to be handled, namely 1) No Horn Zone, 2) Parking Zone, 3) Obstacle Detection.

1.No Horn Zone: When the vehicle enters in the normal zone its horn does not disabled and it goes normally no action is performed. When the vehicle enters into the restricted areas that means it enters into the No-Horn Zone the horn is disabled.

2.Parking Zone: In Parking Zone,We are using the GPS module it specifies the particular locationwhether the location is parking zone or not and notifies to the driver about it.

3.Obstacle Detection:We divide the distance between the driver and the obstacle into 3 sections, the first $8 \mathrm{mts}$ from the vehicle to obstacle is considered as safe zone where no action is taken, the next $5 \mathrm{mts}$ from the vehicle to obstacle is considered as caution zone, where the caution is displayed to the driver, at last when the vehicle nears to $3 \mathrm{mts}$ from the obstacle, it is a danger zone where the driver is notified through abuzzer. This mechanism is achieved using IR-Sensor.

\section{Expected Results}

This system aims to ensure drivers' safety. The system also helps to limit the speed of the vehicle thus by reducing the risk of accidents. The data are all stored in database that are present in the cloud are all verified and validated. When the testing is done and executed it shows a latitude, longitude of the current location of the moving vehicle, distance covered by the vehicle,address of that location and also the rules that are fetched from the cloud.

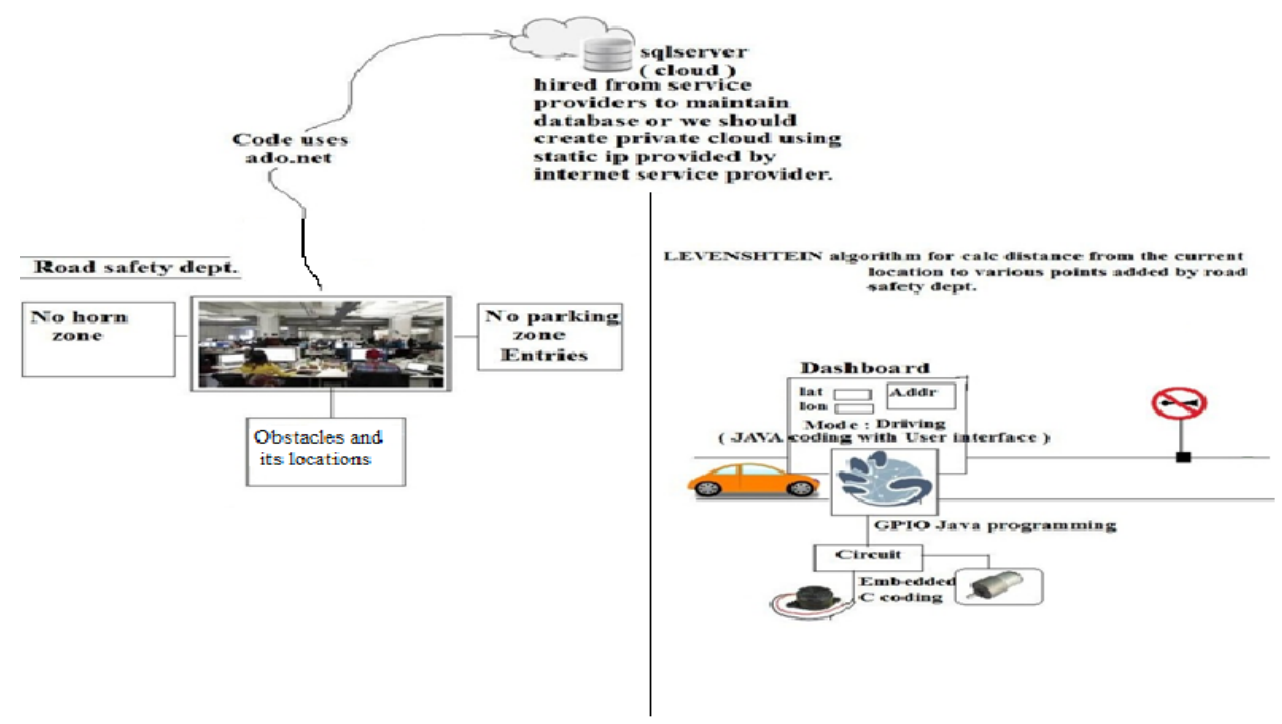

Fig 1: System Architecture

Proceedings of the $3^{\text {rd }}$ National Conference on Image Processing, Computing, Communication, Networking and Data Analytics (NCICCNDA 2018) 
Road Safety Surveillance and Intelligent Traffic Management System

\section{Conclusion}

This system presents a real time road safety monitoring to solve the problems caused due to breaking of rules. The proposed system provides a new way to control traffic rules. The traffic administration department can use this real time roadsafety monitoring system to detect dangerous situations on the road and thereby react by imposing immediate actions. On the whole IOT will play an important role in road safety monitoring system.

\section{Future Work}

- In the future we can try to implement the application in system for controlling traffic signals. That is, we can make the vehicle to stop by any means of obstacle when the signal turns red, and allowing the vehicle to move when it turns green.

- Enhancement of gesture control. By introducing a new feature called MMI (multimedia touch) that would provide un precendented ease in controlling a vehicle's functions.

- Communication between vehicles: Communication between vehicles mean y machines communicating with each other so that there'll be smoother traffic and less congested roads.

- Smart Fuel Saving Tips: Another feature to have in a smart car is to have it give your fuel efficiency tips or notifications while you're driving. For ex it can notify you about a nearby gas station that has cheap gas prices.

\section{References}

[1] J. Sherly1, D.Somasundareswari Scholar, ECE Department, SNS College of Technology, Tamilnadu, India, "Internet of things based smart transportation systems"IRJET, vol. 02, issue. 07, 2015.

[2] S.P. Bhumkar, V.V. Deotare, R.V.Babar,Sinhgad Institute Of Technology, Lonavala, Pune, India “Accident avoidance and detection On highways" IJETT, vol. 03, issue. 02, 2012

[3] M. Zimmer, PhD Candidate, Department of Culture and Communication, New York University "Surveillance, privacy and the ethics of vehicle Safety communication technologies", vol. 07, issue. 04, 2005.

[4] ManaliRaje, Maharashtra Institute of TechnologyPune, India, "Algorithm for Back-up and Authentication of Data Stored on Cloud", 2015.

[5] http://ijarece.org/wp-content/uploads/2016/06/IJARECE-VOL-5-ISSUE-5-1615-1618.pdf

[6]. http://www.ijsrp.org/research-paper-1215/ijsrp-p4898.pdf

[7]. https://www.irjet.net/archives/V2/i7/IRJET-V2I7196.pdf

[8]. https://www.rospa.com/rospweb/docs/advice-services/road-safety/vehicles/black-box technology.pdf 